Association for Information Systems AIS Electronic Library (AISeL)

ECIS 2000 Proceedings

European Conference on Information Systems

2000

\title{
Internet Newspapers: Are Some More Equal than Others?
}

Enrique Dans

UCLA, enrique.dans@anderson.ucla.edu

Follow this and additional works at: http://aisel.aisnet.org/ecis2000

\section{Recommended Citation}

Dans, Enrique, "Internet Newspapers: Are Some More Equal than Others?" (2000). ECIS 2000 Proceedings. 94.

http://aisel.aisnet.org/ecis2000/94

This material is brought to you by the European Conference on Information Systems (ECIS) at AIS Electronic Library (AISeL). It has been accepted for inclusion in ECIS 2000 Proceedings by an authorized administrator of AIS Electronic Library (AISeL). For more information, please contact elibrary@aisnet.org. 


\title{
Internet Newspapers: Are Some More Equal than Others?
}

\author{
Enrique Dans \\ The Anderson School of Management at UCLA \\ 110 Westwood Plaza, Suite D501 \\ Box 951481 \\ Los Angeles, CA 90095-1481 \\ Enrique.Dans@anderson.ucla.edu \\ and \\ Instituto de Empresa \\ Madrid, Spain
}

\begin{abstract}
In 450 years of existence, the written press has never faced a change with the intensity and consequences as those of the Internet. The decision of going online represents a whole set of opportunities and threats that publishers must carefully weigh. Once they have made the step, newspapers find themselves in a completely different competitive environment. They deal with different competitors, business models, customers and patterns of consumption. The present study explores such patterns of consumption by using data from 15 Spanish newspapers, covering time periods from 2 to 20 months. Characteristics of the printed newspapers and general patterns of the Internet are also analyzed in order to isolate the factors that enable an Internet newspaper to achieve a high level of readership. The findings indicate that reading patterns on the Internet strongly differ from those in the physical world, particularly when we consider weekday versus weekend circulation. The use of the newspaper is different too: reading of Internet newspapers is usually more functional and goal oriented, as indicated by the small number of pages read per visit. These and other results can provide publishers with valuable insights to understand this new frontier in the history of journalism.
\end{abstract}

\section{INTRODUCTION}

The earliest antecedent of newspapers is considered to be the avvisi or fogli a mano, manuscript pieces of news born in Venice in the XV century. Although not completely periodical, the avvisi were a valuable resource used by bankers and merchants to get information about the changing sociopolitical environment of that time. It was an exclusive and expensive service, and the journalists (menanti) were influential and well-known people. With the advent of the printing press, the situation changed: it was easy to print a large number of copies of a particular piece of news, so the original avvisi became the gazetta, so called following a small Venetian coin that was its price. However, manuscript and printed news coexisted during throughout the XVI century and beyond. The coexistence of these two different forms is just one example of how slowly established institutions surrender their place to new ones - even when the new ones are better. The invention of the telegraph in 1837 and the telephone in 1875 are further examples in which a significant technological breakthrough affected the way journalism was pursued. These innovations represented initially expensive options adopted by major newspapers, but turned into a must a few years later. The Internet is another innovation with the potential of changing the way we understand newspapers today. It has the potential of changing the cost structure of a newspaper, traditionally dominated by the cost of tangible elements such as paper and ink, and to greatly diminish the cycle time for delivering the news. Such changes motivate newspapers to compete in new ways: new strategic models include competition on speed, cost or differentiation.

The number of newspapers available on the Internet has been steadily growing over the past two years. According to E\&P Interactive Online Newspaper Database, the number of newspapers in the world with an Internet edition was 3,394 in February 1999. In most developed countries, almost all of the major newspapers either have an online presence or are currently in the process of implementing projects for doing so. One important reason for this phenomenon is the exponential growth of the Internet, which turns it into a medium whose importance cannot be neglected. Second, the habit of reading the news online appears to be an increasing trend, so publishers are afraid that if they don't go online as soon as possible, they will lose their readers to other competitors. Third, most newspapers can afford the initial investment required to set up an electronic edition, since most of the cost has already been invested to create the printed version. Finally, the Internet offers a variety of funding opportunities: subscription fees, advertising, pay per use, and can serve as a highly efficient medium for classified advertising, a major source of revenue for most newspapers.

The present study addresses the question of the consumers' response to Internet newspapers, and how this response is influenced by the characteristics of these newspapers in the physical world. To do so, characteristics of the printed newspapers such as size, reading patterns or demographic profiles of the readers are analyzed along 
with characteristics of the Internet, and a contingency framework is used to analyze to what extent characteristics of the newspapers match those of the Internet itself. As the title of the paper suggests, some newspapers appear to be more equal to the Internet profile than others.

The remainder of the paper is organized as follows. The next section reviews the relevant literature and introduces a theoretical framework for the study. Section 3 briefly discusses the data and outlines the methodology employed for the analysis. Section 4 presents the results obtained, which are then discussed in Section 5. Section 6 concludes the article, highlights potential limitations and suggests directions for future research.

\section{THEORY}

Some characteristics of Internet newspapers have been cited in recent IS and marketing literature. The good fit between newspapers and the Internet is advanced by Rayport and Sviokla [19], depicting the Internet as an ideal distribution channel for goods consisting of digital assets, because the variable cost of distributing them is nearly zero. Peterson et al. [16] provide a framework for classification of products and services in the context of the Internet into three relevant dimensions: cost and frequency of purchase, value proposition and degree of differentiation. A product such as a newspaper is classified as a low outlay, frequently purchased good, with a value proposition of intangible or informational nature, and a high differentiation potential. According to Peterson et al. [16], these are attributes that ensure a great advantage in using the Internet as a transaction and distribution channel. The same authors draw a likely decision sequence for online newspapers and magazines based on these attributes: first, the consumer is expected to choose a brand after an Internet search. Second, a subsequent price search in retail channels is very unlikely. Third, the final acquisition of the good occurs most likely on the Internet. This consumer decision sequence leads the authors to believe that the editorial sector will be strongly affected by the emergence of the Internet.

Other scholars take the emergence of electronic newspapers for granted. Negroponte [13], for example, envisions a future in which all news will be conveyed in digital format, and the associated challenge will not be related to bandwidth, but rather to finding ways to filter and classify huge amounts of information. Watters et al. [21] admit that there is some concern about the acceptability of the newspaper in electronic versus paper form, but forecast that the electronic form appears to be inevitable. They apply two behavioral theories to news reading: the uses and gratification [6] and the ludenic or play [20]. The first approach considers a user with some goal or task to accomplish that must be satisfied by getting the news. The second theory suggests that the process of reading the news is intrinsically pleasurable, leading to a more casual, spontaneous and unstructured form of news reading. Readers can follow the two approaches simultaneously, switch back and forth between them or not use them at all; the final outcome depends on a number of factors. For instance, Bogart [2] demonstrated that people read the weekday paper and the weekend paper in a different way: the weekend paper has a much longer average lifespan at home, and it is generally read more carefully, deploying a longer time to do so in a more relaxed way. According to our hypotheses, these differences in readership patterns will occur also in Internet newspapers.

Palmer et al. [15] draw a set of very interesting comparisons between printed and Internet newspapers by thoroughly analyzing 48 of them. The authors define a model in which both the technological features of the Internet newspapers and their marketing implications converge in a specific Internet newspaper design, which obtains a certain market response. However, the article focuses on the characteristics of the newspapers, but does not analyze the way consumers react to them. Such market response and its characteristics are precisely the subject of our study.

Another interesting issue emerges from the supposed global scope of the Internet. According to Quelch and Klein [17], any company that establishes a site on the Internet automatically becomes a multinational company, because consumers worldwide can access it. However, newspapers are normally national, regional or even local products. In reality, very few newspapers trespass beyond national borders, with exceptions being papers such as The Wall Street Journal and the Financial Times. Jarvenpaa and Todd [9] follows this line of reasoning: contrary to "common wisdom", all sellers are not equal on the Internet, where previous knowledge and reputation are crucial for attracting customers.

Other authors are more cautious about the Internet hype. Kalakota [10] describes the efforts of some publishers for developing an online edition as a "Just get me on the web!" approach. According to these authors, the early phase of online publishing, from 1993 to 1995, is characterized by a complete lack of business purpose and web sites created by techno-savvy individuals who did not understand the business of publishing. Brännback [3] also portrays this lack of business objectives and its possible effects: "the inability to understand the difference between competing in the marketplace vs. the marketspace can cause more damage than anticipated."

Following Orlikowsky and Yates [14], a newspaper can be viewed as a "communication genre" enacted either by a traditional medium or "carrier" such as paper, or by a new carrier such as the Internet. A commu nication genre is defined as "a socially recognized type of communicative action that is habitually enacted by members of a community to realize particular social purposes" [23]. 
While the determinants of consumers' responses have been well documented and studied over the years for the traditional carrier, the way consumers respond to the enactment of the newspaper genre via a new carrier remains uncertain. The Internet is a new medium in which the characteristics of consumers' responses are not yet clearly established.

Viewed from a contingency perspective, this consumer response is conditioned by the level of fit between properties of the newspaper genre and characteristics of its carrier here: the Internet. In this contingency framework, the concept of "fit" is defined from an interaction perspective [7]: variations in organizational performance are explained from the interaction of the organization and the context. Applied to the present case, this means that the more similar a newspaper is to the Internet in terms of demographics, reading patterns or other factors, the better the online version of this newspaper will perform. Hence, organizations should try to achieve such fit, or, in other words, should try to become isomorphic with their contingency approach, this hypothesis attempts to establish the baseline change that affects all newspapers when they move from the physical world to the Internet, driven by the differences in size between both markets.

Hypothesis 2: The higher the degree of similarity in demographic profile between newspaper's readers and Internet users, the more readers will be attracted to the newspaper's Internet edition.

Consistent with the notion of fit (see Figure 1), this hypothesis tests to what extent differences in the distribution of gender, age, socioeconomic status (SES) and level of education between newspaper's readers and Internet users affect how that particular newspaper appeals to the newspaper's Internet readers. We expect newspapers with a closer profile to that of the Internet to get a significantly higher number of readers in its Internet edition, once the effect of size has been discounted.

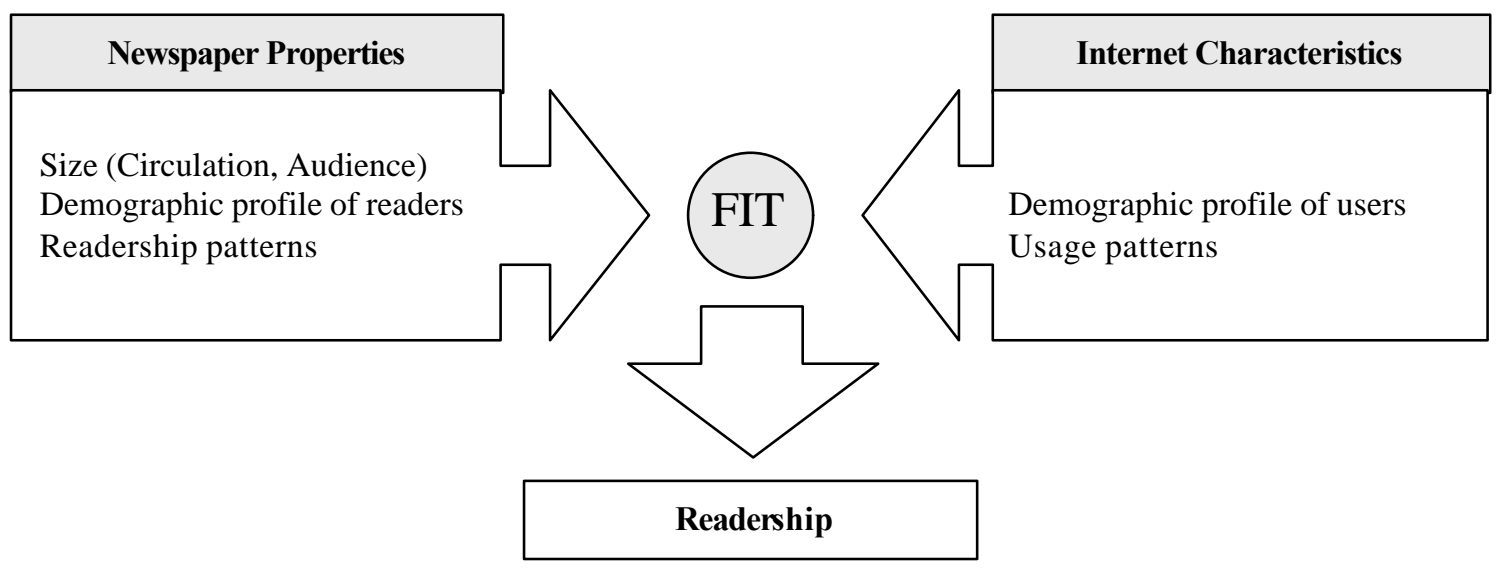

Figure 1: Research model

normative environment, increasingly resembling their environment and each other over time [5]. This study attempts to identify a set of "ideal characteristics" and to measure their influence in getting a highly read Internet edition (see Figure 1)

According to this theoretical framework, the following hypotheses can be formulated:

Hypothesis 1: The larger the size in terms of circulation or audience of the printed newspaper, the more readers will be attracted to its Internet edition.

This hypothesis takes into account that the access to the Internet is not universal yet. It attempts to determine to what extent the size of the newspaper expressed in number of readers or in number of copies sold affects the number of readers it gets in its electronic edition. In our
Hypothesis 3a: For Internet newspapers, weekend readership will be lower than weekday readership.

Hypothesis 3b: For Internet newspapers, the decline in weekend readership will be more pronounced than that of the rest of the Internet.

Printed newspapers usually increase their circulation approximately $50 \%$ on weekends. According to our hypothesis, Internet newspapers would, however, behave exactly in the opposite way as printed newspapers do. Two effects might have an influence here: first, general Internet consumption patterns signal a downward bias on weekends versus weekdays, partially attributable to users who do not have a computer at home. Second, there is a potential substitution effect between the printed and the Internet version of the newspaper: the weekend edition of 
the printed newspaper is so appealing to readers, that many people would choose to read it instead of the Internet edition, even when having a connection at home.

Hypothesis 4: The number of pages per visit read on Internet newspapers during the weekends will be higher than during the weekdays.

Consistent with [2], we expect a use of the Internet newspaper closer to the ludenic or play theoretical approach during the weekends and more functional and related to the uses and gratification approach during the weekday. This more relaxed way of weekend reading should be reflected in a higher number of pages per visits read during the weekends, following the same pattern as the paper world.

The motivation for the five hypotheses is duofold: Hypotheses 1 and 2 should allow for an understanding of the factors driving online readers to a particular newspaper. Hypotheses $3 \mathrm{a}, 3 \mathrm{~b}$ and 4 , should provide a framework to understand the magnitude and causes of the weekend effect. As discussed later, knowledge of these two issues may provide interesting insights to both researchers and practitioners in the field. six national, six regional and three provincial papers (see Table 1 for descriptive data). Most are general newspapers, although the sample also includes the two leading economic and the main sports newspaper. The data collection was restricted to electronic newspapers audited by OJD in order to use a consistent and widely accepted method for accounting for visits. Electronic newspapers not audited by OJD do not keep track of their number of visits or base their figures on internal accounting methods. Internal accounting methods cannot always be trusted, since advertising revenues are a function of the number of visits and pages. In the absence of a commonly accepted method for accounting for visits, these newspapers would be obviously interested in artificially inflating the number of visits and pages, thus becoming more attractive to potential advertisers.

Data for the electronic newspapers include number of daily visits to the newspaper's site and number of pages read daily. Additionally, we collected data from Olé (http://www.ole.es), the most popular Spanish search engine, to characterize the Internet consumption patterns in Spain. The study also uses data from the paper edition of the newspapers: circulation (number of copies sold),

T ABLE 1: DESCRIPTIVE DATA OF THE NEWSPAPERS IN THE SAMPLE

\begin{tabular}{|c|c|c|c|c|c|}
\hline Nameplate & Geographic Ambit & Type & Circulation" & Audience" & Controlled period \\
\hline La Vanguardia & Regional & General & 210,012 & 640 & May 97-Dec 98 \\
\hline El Correo Gallego & Provincial & General & 16,507 & N/A & May 97-Dec 98 \\
\hline Canarias 7 & Provincial & General & 36,796 & 166 & Jun 97-Dec 98 \\
\hline El Diario Vasco & Provincial & General & 93,553 & 344 & Jul 97-Dec 98 \\
\hline El Mundo & National & General & 284,519 & 926 & Sep 97-Dec 98 \\
\hline $\mathrm{ABC}$ & National & General & 301,054 & 952 & Dec 97-Dec 98 \\
\hline El Pais & National & General & 440,628 & 1,572 & Jan 98-Dec 98 \\
\hline La Rioja & Provincial & General & 16,064 & N/A & Feb 98-Dec 98 \\
\hline El Periódico de Catalunya & Regional & General & 207,772 & 912 & Mar 98-Dec 98 \\
\hline Expansión & National & Economic & 48,170 & 133 & Jun 98-Dec 98 \\
\hline Marca & National & Sports & 458,441 & 2,440 & Jun 98-Dec 98 \\
\hline Levante & Regional & General & 53,676 & 349 & Jul 98-Dec 98 \\
\hline Cinco Días & National & Economic & 26,655 & N/A & Nov 98-Dec 98 \\
\hline Diario de Navarra & Provincial & General & 63,212 & 228 & Dec 98-Dec 98 \\
\hline Avui & Regional & General & 34,156 & 138 & Nov 98-Dec 98 \\
\hline
\end{tabular}

\section{DATA AND METHODOLOGY}

\section{A. Data Collection}

The present study analyzes data from 15 Spanish Internet newspapers audited by $\mathrm{OJD}^{1}$, in periods ranging from 20 to 2 months. The examined newspapers include

\footnotetext{
${ }^{1}$ OJD (Organización para la Justificación de la Difusión) is the Spanish equivalent to the Audit Bureau of Circulation. OJD is a not-for-profit association whose objective is to provide trustworthy data about distribution and diffusion of periodic publications. OJD audits 85 newspapers $(96 \%)$ in Spain.
}

audience (estimated number of readers), and demographic profile. This last variable is measured along four dimensions: age, gender, socioeconomic status (SES) and level of education. Finally, data about Internet usage and growth are gathered too, including growth during the examined period and demographic profile of the users (age, gender, SES and level of education).

The instrumentation and metrics of the variables are the following (see Table 2 for a summary of descriptive statistics)

Visits: The number of visits to the electronic newspaper. Logarithmic transformation was performed to 
eliminate the asymmetries caused by the large differences in size among the observational units, which could bias the results by giving too much weight to the observations of the big newspapers. A visit is defined as an uninterrupted sequence of page impressions performed by a user in a web site. measure for each newspaper in relation to the average Internet profile ${ }^{2}$.

Weekly patterns: A set of dummy variables, one for each day of the week, was created to test the existence of weekly patterns in the evolution of the number of visits, pages and pages per visit.

TABLE 2: SELECTED DESCRIPTIVE STATISTICS

\begin{tabular}{lrrrrrrr}
\hline & Observations & \multicolumn{2}{c}{ Visits" } & \multicolumn{2}{c}{ Pages } & Pages & Demographic \\
\cline { 6 - 7 } Nameplate & & Average & St. Dev. & Average & St. Dev. & Visits & Profile** \\
\hline La Vanguardia & 593 & 3,799 & 1,509 & 22,632 & 12,148 & 5.96 & 61.68 \\
El Correo Gallego & 600 & 207 & 68 & 872 & 274 & 4.21 & N/A \\
Canarias 7 & 546 & 582 & 153 & 2,214 & 731 & 3.80 & 90.36 \\
El Diario Vasco & 523 & 967 & 451 & 6,983 & 2,853 & 7.22 & 84.5 \\
El Mundo & 468 & 20,360 & 7,249 & 128,498 & 50,752 & 6.31 & 53.33 \\
ABC & 388 & 8,951 & 2,835 & 38,200 & 11,326 & 4.27 & 62.1 \\
El Pais & 339 & 42,109 & 14,438 & 300,320 & 97,358 & 7.13 & 55.7 \\
La Rioja & 334 & 229 & 97 & 2,166 & 962 & 9.46 & N/A \\
El Periódico de Catalunya & 282 & 4,089 & 1,312 & 56,575 & 17,209 & 13.84 & 76.81 \\
Expansión & 207 & 7,116 & 3,509 & 36,277 & 15,831 & 5.10 & 53.58 \\
Marca & 209 & 11,428 & 4,485 & 87,274 & 57,568 & 7.64 & 81.77 \\
Levante & 166 & 878 & 430 & 13,851 & 7,580 & 15.78 & 76.05 \\
Cinco Días & 48 & 6,405 & 4,215 & 38,858 & 21,118 & 6.07 & N/A \\
Diario de Navarra & 31 & 715 & 279 & 4,403 & 1,906 & 6.16 & 89.33 \\
Avui & 61 & 1,752 & 487 & 24,825 & 7,730 & 14.17 & 63.84 \\
\hline
\end{tabular}

* Data from OJD

** Data from AIMC, transformed

Pages: The number of pages viewed at the electronic newspaper. A page or page impression is defined as the combination of one or more files presented to a user as a single document as a result of a single request received by the server. Logarithmic transformation was also performed for the same reason as for visits.

Internet activity: Instrumented by the logarithm of the number of visits to Olé.

Circulation: Number of copies sold of a printed newspaper. It is audited by OJD and reported quarterly. As in previous variables, the dissimilarities in the sizes of the newspapers called for logarithmic transformation.

Audience: $\log$ of the number of readers of a printed newspaper, accounting for the fact that each copy is in most of the cases read by more than one person. It is measured by AIMC using a survey instrument administered quarterly.

Demographic profile: Demographic profiles for the printed newspapers and for the average Spanish Internet user were obtained from the AIMC survey, covering gender, SES, level of education and age distributions. The measure for SES was expressed in a five points scale (low, average-low, average, average-high and high). Level of education was measured in a seven points scale following the Spanish educational system, while age employed a seven intervals classification. Global proximities were calculated by generating an Euclidean dissimilarity coefficient matrix, thus generating a unique distance
Vacation: A dummy variable was created to account for the low activity periods in Spain, typically August and Christmas.

\section{B. Data Analysis}

A panel data model is used to combine cross-section and time series data. The motivation to use a panel data approach is to be able to combine the time-series analysis with the cross-sectional approach, taking advantage of a larger number of observations ${ }^{3}$. In the panel data model there are $\mathrm{N}$ cross-sectional units, in our case newspapers, that are observed over $\mathrm{T}$ time periods that span from May 1, 1997 to December 31, 1998. Two panels were generated: the first one, intended to test Hypotheses 1 and

\footnotetext{
${ }^{2}$ A unique measure was preferred to reflect the overall similarity between each newspaper's characteristics and those of the Internet. Since the demographic data was introduced as a set of constants (demographic data do not change, or very slightly, during our twenty month study), a unique measure was proved to be more appropriate for our panel data approach than a set of multiple measures.

${ }^{3}$ A compendium of the earliest literature is [11]. Surveys on the econometrics of panel data include $[8,4,12,18,1]$. The advantages of panel data are described in detail in [8], Ch. 3 and [1]. These authors state that the main motivation for Panel Data is to control for unobserved heterogeneity and for inter-temporal variation. Other well-known advantages are: getting rid of multicollinearity in the data of ordinary models like OLS; taking advantage of more observations NxT and therefore more degrees of freedom than OLS; and to avoid the omitted variables problems of OLS and those of measurement errors since now we are not using aggregate data (i.e. GDP) but individual measurements by observational unit which are usually more accurate.
} 
2 , uses weekly data about visits and pages read and introduces only the 12 newspapers for which audience data are available. The second panel is designed to test Hypotheses 3a, 3b and 4, and disaggregates the weekly data into daily data for all 15 newspapers to measure effects associated with the day of the week. This yields two unbalanced panels with $\mathrm{N} \times \mathrm{T}$ observations. In the first case, with weekly observations, we have an unbalanced panel of 556 data points. In the second case, with daily data, the unbalanced panel has 4,795 data points. The missing observations correspond mainly to the irregularity of the controlled periods for each newspaper (see Table 1) and, in some isolated cases, to technical problems in the newspapers' servers.

The panels were estimated using Seemingly Unrelated Regression (SUR), due to the existence of both heteroskedasticity and contemporaneous correlation among the cross-sectional units ${ }^{4}$. Other alternatives for estimation such as OLS and Weighted Least Squares (WLS) were also tested in order to check for robustness, and yielded parameter values consistent with those for SUR.

\section{RESULTS}

We first generated descriptive statis tics (see Table 2) and correlation matrices among the variables. A preliminary analysis of the individual time series using ordinary least squares (OLS) regression showed the presence of dynamic components, with Durbin-Watson coefficients indicating positive serial correlation, significant at the 0.05 level. The analysis of the correlograms confirmed such serial correlation. The ACF and PAC of the individual newspapers were examined, and significant spikes were found in the first few lags of all the PAC's, while the ACF smoothly died down. A standard ADF unit root test was performed to reject the presence of unit roots in each of the individual series ${ }^{5}$.

For Hypotheses 1 (size) and 2 (demographic profile), the panel is organized as follows:

$\log \left(\right.$ Vis $\left._{i t}\right)=\boldsymbol{\alpha}+\boldsymbol{\beta}_{1} \log \left(\right.$ Aud $\left._{i}\right)+\boldsymbol{\beta}_{2} \operatorname{Dem}_{i}$

$+\boldsymbol{\beta}_{3} \operatorname{Vac}_{t}+\gamma_{i} G r_{i t}+\rho \log \left(\operatorname{Vis}_{t-1}\right)+\boldsymbol{\varepsilon}_{i t}$

\footnotetext{
${ }^{4}$ The seemingly unrelated regression (SUR) method, also known as the multivariate regression, or Zellner's method, estimates the parameters of the system, accounting for heteroskedasticity and contemporaneous correlation in the errors across equations, and it is considered to be the most reliable method under those circumstances [24].

${ }^{5}$ If the existence of a unit root cannot be rejected, the series should be at least first differenced before being used in any statistical model. Time series with unit roots will not usually produce reliable statistical results because asymptotic distributions are never achieved. In our case, an Augmented Dickey-Fuller test including trend and intercept in the equation (the correct instrumentation for the test and the number of augmentation terms included were tested using the Schwarz criterion and the analysis of the individual OLS regressions), rejected the presence of unit roots for all newspapers at the 0.05 level, except in two cases (the two shorter series), in which it was rejected at the 0.1 level.
}

The twelve electronic newspapers are the observational units $(i=1,2, \ldots, 12)$ for the cross-sectional part of the model. Time series of the $\log$ of the number of weekly visits $\left(V i s_{i t}\right)$ were used as a dependent variable. The log of the printed newspapers' audience $\left(A u d_{i}\right)$, proximity in demographic profile $\left(D e m_{i}\right)$, a linear trend to reflect growth $\left(G r_{i t}\right)$, a dummy variable to indicate vacation periods $\left(V c_{t}\right)$ and an auto-regressive coefficient of order one $\left(V i s_{t-1}\right)$ were used as independent variables ${ }^{6}$. The log of the printed newspaper's circulation was used as an alternative measure to audience, in order to check which one achieved better results. A common coefficient was given to the variables that should yield the same parameter across all members of the pool, whereas specific coefficients were assigned to variables needed to display the particular behavior of each observational unit. Growth was calculated as a specific coefficient, while the influence of demographic profiles, vacation periods and newspapers' audiences were estimated using common coefficients. A common intercept $\left(\boldsymbol{\alpha}_{\boldsymbol{i}}\right)$ was estimated, since differences in size were already taken into account by the introduction of the audience or circulation data. The error $\left(\boldsymbol{\varepsilon}_{i t}\right)$ is presumed to have the standard properties of the SUR approach (i.e. heteroskedasticity and contemporaneous correlation).

This panel yielded an $R^{2}$ of 0.97 using SUR estimation ${ }^{7}$. The presence of dynamic components on the panel was confirmed by the initially low value of the Durbin-Watson statistic, 0.71. This value went up to 2.01 when a first-order auto-regressive coefficient was introduced into the equation, as advanced by the analysis of the individual OLS regressions. The size of the printed newspaper, the differences in demographic profile and the effect of vacation periods were estimated as common coefficients, and were found to be significant at the 0.05 level. Growth was estimated individually for each cross-sectional unit. Of the twelve newspapers, eight showed positive growth significant at the 0.05 level, one displayed slightly negative growth, and the remaining three yielded nonsignificant growth coefficients.

When printed newspaper's audience is replaced by printed newspaper's circulation, the results are very similar. The overall panel achieves the same $R^{2}$. The t-statistic for audience is higher, 10.45 versus 8.46 in the case of circulation. The other parameters in the equation remain consistent.

\footnotetext{
${ }^{6}$ Higher order auto-regressive coefficients were also tested. Even though some of them were significant, the increase in explanatory power was marginal, and its introduction was rejected according to the Schwarz criterion.

${ }^{7}$ Such high values of $R^{2}$ are somewhat common when dynamic panel data models are used, due to the parallel evolution of several crosssectional units across time.
} 
The panel for Hypotheses $3 \mathrm{a}$ and $3 \mathrm{~b}$ (weekend effect) observes variables at a daily level and uses all the fifteen newspapers.

$$
\begin{aligned}
& \log \left(\operatorname{Vis}_{i t}\right)=\boldsymbol{\alpha}_{i}+\boldsymbol{\beta}_{1} \operatorname{Vac}_{t}+\boldsymbol{\beta}_{2} G r_{i t}+\sum_{j=1}^{6} \boldsymbol{\delta}_{j} W d_{j t} \\
& +\boldsymbol{\rho} \log \left(\operatorname{Vis}_{t-1}\right)+\boldsymbol{\varepsilon}_{i t}
\end{aligned}
$$

The dependent variable is again the log of the number of visits $\left(V i s_{i t}\right)$, but disaggregated at the daily level. As independent variables, we use a set of dummy variables for each of the days of the week ${ }^{8}\left(W d_{j t}\right)$, another dummy to indicate vacation periods $\left(\mathrm{Vac}_{t}\right)$, a linear trend to reflect growth $\left(G r_{i t}\right)$ and a first-order auto-regressive coefficient $\left(V i s_{t-1}\right)$. The intercepts in this model $\left(\boldsymbol{\alpha}_{i}\right)$ were calculated for each observational unit (fixed effects), since the magnitude of the observational units was very dissimilar. As in the previous panel, the error $\left(\varepsilon_{i t}\right)$ is presumed to have the standard properties of the SUR approach.

This second panel provided an $R^{2}$ of 0.99 using SUR. Again, the Durbin-Watson statistic initially displayed a value of 1.14 , evidence of a strong positive serial correlation, but went up to 1.93 when we introduced a first-order auto-regressive coefficient. The coefficients for the days of the week, estimated as common, were all significant at 0.05 level; Monday, Tuesday and Wednesday showed positive œefficients in relation to Friday, while Saturday and Sunday displayed negative ones. The effect of vacation periods, also estimated as a common coefficient, was significant and negative at 0.05 level. The results for growth, estimated as a specific coefficient for each cross-sectional unit, were consistent with that of the previous panel. For the three newspapers not included in the previous panel, two were significant and positive, and the third was not significant.

For Hypothesis 4 (pages per visit read during the weekend), the panel is similar to the previous one, but using the number of pages divided by the number of visits for both dependent and independent variable. Once again, the intercepts were calculated as fixed effects for each observational unit, since the number of pages per visit is likely to be specific for each newspaper and dependent on factors such as the design of the pages and the structure of the newspaper (see Table 2). In this case, the panel rendered an $R^{2}$ of 0.83 , also using SUR estimation. The coefficients for the days of the week showed no significant differences among Tuesday, Wednesday and Friday. Monday and Thursday, in contrast, showed negative coefficients for the number of pages read per visit. The coefficients for Saturday and Sunday were significant and positive, 0.16 and 0.28 respectively.

\footnotetext{
${ }^{8}$ Friday was left out because it proved to be the average day of the week for most newspapers in the individual OLS regressions performed.
}

Finally, the coefficients obtained for the days of the week were compared to the ones obtained in an individual OLS regression performed on Olé using the same set of parameters as the original panels. The number of visits for Olé did not show significant differences among Mondays, Wednesdays, Thursdays and Fridays. The number of visits for Tuesdays was slightly higher, and those of Saturdays and Sundays were significantly lower, although not as low as the ones displayed by the newspapers $(-0.28$ and -0.31 in Olé versus -0.49 and -0.45 in the newspapers' panel for Saturdays and Sundays respectively). Regarding pages per visit, no statistically significant differences were found from Monday through Saturday in Olé, while Sundays displayed a lower number of pages read per visit, -0.12 .

\section{DISCUSSION}

The findings of the study support the proposed hypotheses. The effect of the printed newspaper's size on the number of visits is supported for both measures of newspaper size, circulation and audience. In the panel designed to test this hypothesis, the elasticity of the visits with respect to both circulation and audience was 0.75 . Additional support for Hypothesis 1 is provided by the observed correlation between the fixed effects obtained in the panel with daily data and the size of the newspapers ( 0.78 for circulation and 0.70 for audience). Accordingly, as a general proposition, larger printed newspapers should tend to attract a higher number of visitors to their electronic editions.

The introduction of the similarity of profiles into the equation also yields the expected results: newspapers with demographic profiles close to that of the Internet consistently get a higher number of visits than expected considering their size. The most salient case occurs with the two economic newspapers in our sample: even though their circulation is among the smallest in our sample, they get a number of visits much higher than expected. The profile similarity between economic newspapers and the Internet is evident in the four aspects measured: gender, SES, age and level of education. The sports newspaper in the sample represents the other extreme: being the largest Spanish newspaper overall in both circulation and audience, the number of visits is much lower than expected, consistent with the big differences between its demographic profile and that of the Internet.

The effect of the weekend on the number of visits to electronic newspapers can be characterized as a severe drop: $46 \%$ and $40 \%$ fewer visits respectively on Saturday and Sunday in comparison to the average weekday. The number of visits during the weekdays remains approximately stable, with variations between $2 \%$ and $8 \%$. Monday appears to be the best day of the week, being an 8\% larger than Friday. Between them, there is a gradually descending trend: In comparison to Friday, Tuesday has $7 \%$ more, Wednesday reduces the difference to $5 \%$ and 
Thursday is just $3 \%$ more. Contrasting with this relatively stable intra-week pattern, visits drop to about a half during weekends. The reasons for this pronounced plunge are probably twofold: people who do not have an Internet connection at home and buyers of the weekend edition of the printed newspaper, which becomes a true substitute for the Internet newspaper. Regarding the effect of the lack of Internet connection, however, it is important to note that when the complete panel was split into two, the weekend effect remained almost the same, being in fact a little more pronounced in the second half than in the first one. During our controlled period the number of Internet users in Spain almost doubled (3.9\% to $7.1 \%$ over the adult population) and the number of homes equipped with a computer grew from $19 \%$ to $24 \%$. These two facts cast some potential doubts about the responsibility of the lack of home connectivity for the weekend effect.

The decline in readership associated with the weekend is also more pronounced in newspapers than in the rest of the Internet, thus supporting Hypothesis $3 \mathrm{~b}$. The individual OLS performed on Olé yields a $28 \%$ and a $31 \%$ decrease in visits over Saturday and Sunday respectively, substantially smaller than the figures observed in electronic newspapers. The patterns for the rest of the week are also different in Olé: there are no significant differences among the weekdays, except for Tuesday, which enjoys a $7 \%$ increase in visits. Therefore, the existence of such a pronounced weekend effect is characteristic and specific to Internet newspapers, thus differentiating them from the average patterns of Internet usage. Therefore, electronic newspapers can be considered a new and differentiable way to enact the newspaper genre [14], as postulated in our theoretical framework.

Hypothesis 4 provides additional insights into understanding the reasons behind the weekend decline in readership. The results of the panel when pages per visit substitute for visits as a dependent variable lend support to the proposed hypothesis: there are almost no significant differences in the number of pages read per visit during the weekdays. Monday shows a slightly superior coefficient in relation to Friday, $0.08 \%$, while Thursday behaves in the opposite way with a slight decrease, $-0.06 \%$. The weekend, in contrast, displays a remarkably different pattern, with strong positive coefficients of $16.1 \%$ and $28.2 \%$ for Saturday and Sunday respectively. This pattern remains stable for the controlled period. This finding replicates the conclusions made by Bogart [2] for printed newspapers: people read the weekday paper and

\footnotetext{
${ }^{9}$ Data from AIMC (Asociación para la Investigación en Medios de Comunicación). Data from AIMC are a good complement of those from OJD, since the first uses auditing methods (physically counting the number of newspapers printed or sold) while the second uses survey methods to estimate audience, etc. AIMC is considered to be the best source in Spain for data about media trends, public consumption and other magnitudes.
}

the weekend paper in a different way, with different approaches and different objectives. Thus, the weekday would characterize the uses and gratification approach, a conscious search for useful information, while the weekend reading would be representative of the ludenic or play approach, more leisure-oriented, relaxed and driven by plain curiosity.

\section{CONCLUSION}

The present study offers new evidence and insight into the consequences for newspapers of venturing into the Internet. It establishes the influence of some characteristics of the newspaper -size and demographic profile- on the number of visits, and illustrates how reading patterns of the newspapers and usage patterns of the Internet interact to create a new pattern, different from both of them and completely specific to Internet newspapers. The study characterizes the effect of weekends on electronic readership. On the one hand, fewer readers are attracted to the weekend Internet edition of the newspaper than during the week, probably due, among other reasons, to the substitution pressure exerted by the popularity of the printed edition. On the other, those readers who do choose the Internet edition on weekends tend to read more pages, imitating the way they read the weekend's printed edition.

The implications for managers are straightforward: first, take into account the way the previous image and characteristics of the newspaper determines its fit in relation to the Internet. This set of characteristics acts as a given when the newspaper decides to venture into the Internet, conditioning the size of the potential audience. The second implication is related to weekly patterns of consumption, and to the way the pre-existing patterns from the physical world interact with those of the Internet. A careful study of these patterns is essential for demand analysis and prediction: publishers could use such studies to decide upon when to place advertising and how to price it, when to increase featured articles or series to improve readership in specific days, or how to design marketing strategies without compromising the quality of service.

Some of these implications might be extended to other products venturing into the Internet. For instance, products, e.g. electronic banking, with a previous image and customer base in the physical world could benefit from the suggestion of comparing the profiles of their current customers with the average Internet profile. They could also try to predict level of usage by analyzing their current patterns of consumption and comparing them with the effects mentioned in this study, such as the weekend effect or the vacation effect.

It is also an intention of this study to shed some light into this interesting period in the history of journalism. The consequences of the Internet as a new medium for newspapers are still unknown, and could range from being 
just another vehicle for accessing the same information, to becoming a completely different product, with elements of convergence with other media such as radio or TV. The possibilities of the Internet to convey image or sound are still being developed, but they do not appear to be very far off. To this extent, the "newspaper of the future" could be a much more dynamic medium, with several or continuous updates during the day, reporters equipped with digital cameras sending real-time, rich-media information, etc. Many newspapers have started to move this way: El Mundo, for instance, offers real-time coverage of some soccer matches, delivering comments to a web page that is updated every minute. El Correo Español - El Pueblo Vasco, the main newspaper in the Basque country, adds the radio version of the news to the regular written news. These could be isolated examples, but they could also be signaling the beginning of a new, changing horizon for journalism. The consequences of such convergence on other media are uncertain. From this viewpoint, this study represents an attempt to portray the moment in which newspapers were faced with this new medium, and the way their customers reacted to it.

Some potential limitations of the study are related to its country-specific nature. In Spain, the growth of the Internet is being slowed by the price structure of telephone communication. The absence of a flat rate for local calls and the high line base rates, for instance, are likely to deter some users from accessing the newspapers on weekends, since they have to cover the cost of the telephone call and the telephone line is kept busy. It would be interesting to get data from other countries and to ascertain how some country-specific patterns affect Internet usage. Additionally, distribution via subscription is extremely low in Spain versus other countries: it would be interesting to check whether the patterns found in the present study apply also to other markets in which a large proportion of readers get the newspaper directly delivered to their home every day. Other limitations of the study arise from the availability of data, particularly in the number of crosssectional units. However, the lack of homogeneity in the measurement criteria and definitions used prevented us from using data from other newspapers not audited by OJD, as previously mentioned in the Data Collection section. Regarding data availability, we must take into account that the present study uses only data aggregated to the level of newspaper. A whole set of interesting research opportunities could arise from the use of individual consumers' data, by means of cookie technology ${ }^{10}$ or similar methods. The analysis of individual data could yield interesting conclusions regarding the usage of the

\footnotetext{
${ }^{10}$ Cookie technology allows the tracking of individual users by placing a small file in their computers. Its use is widespread to store options, passwords or other sorts of data.
}

newspaper by different clusters of users, such as expatriates, weekday readers or weekend readers.

Another interesting issue emerges from the relationship between the traditional, paper newspaper and its electronic counterpart. Although most electronic newspapers are intended to complement, rather than substitute for the physical newspaper, there is undoubtedly a risk of cannibalization. This risk is increased by the fact that most electronic newspapers are for free, and their revenue generation mechanisms are still at a very early stage in most of the cases. A particularly salient case is El País, the largest general newspaper in Spain, which has been forced to close its international edition due to the consumers' shift to the electronic edition. Cases like this draw attention to a phenomenon that requires further research.

A final observation emerges from the issue of the universal access to the Internet: while some authors consider it a valid working assumption [16], present access is clearly not as widespread as it is likely to be in a few years from now. How will this lack of universality affect our conclusions? Undoubtedly, the relative sizes of both markets will change but other characteristics such as the effect of weekends are more likely to persist. An adequate knowledge of such characteristics will be key to understand the future dynamics of competition in this medium.

\section{REFERENCES}

[1]Baltagi, B. "Econometric Analysis of Panel Data". New York: John Wiley and Sons, 1995

[2]Bogart, L. "The State of the Industry", in P. S. Cook, D. Gomery and L. W. Lichty (Eds.) The Future of News\}, Washington, DC: The Woodrow Wilson Center Press, 1992, pp. 85-103

[3]Brännback, M. "Is the Internet Changing the Dominant Logic of Marketing?" European Management Journal v.15, n.6 (Dec 1997) pp.698-707

[4]Dielman, T. "Pooled Cross-Sectional and Time Series Data Analysis". New York: Marcel-Dekker, 1989.

[5]DiMaggio, P., and W. Powell, "The Iron Cage Revisited: Institutional Isomorphism and Collective Rationality in Organizational Fields" American Sociological Review, 48(1983) pp. 147-160

[6]Dozier, D. and R. Rice "Rival Theories of Electronic Newsreading", in R. Rice (ed.), The New Media, (pp. 103-128). London: Sage Publications, 1984

[7]Drazin, R. and A. Van de Ven, "Alternative Forms of Fit in Contingency Theory" Administrative Science Quarterly v.30, n.4 (Dec 1985) pp. 514-539

[8]Hsiao, C. "Analysis of Panel Data" Econometric Society Monographs, \\#11. Cambridge University Press, Melbourne, 1986

[9]Jarvenpaa, S. and P. Todd. "Is There a Future for Retailing on the Internet?" Electronic Marketing and the Consumer, 1997.Ed. Robert A. Peterson, Thousand Oaks, CA. Sage, 139-154

[10]Kalakota, R. and A. Whinston, "Frontiers of Electronic Commerce" Reading, Mass.: Addison-Wesley, 1996 
[11]Maddala, G. "The Econometrics of Panel Data" Vol. 1 and 2. Brookfield, VT: E. E. Elgar, 1993

[12]Matyas, L. and P. Sevestre, eds. "The Econometrics of Panel Data: Handbook of Theory and Applications", 2nd ed. Dordrecht: Kluwert-Nijoff, 1996

[13]Negroponte, N. "Being Digital", New York : Knopf, 1995 [14]Orlikowsky, W. and J. Yates "Genre repertoire: The Structuring of Communicative Practices in Organizations" Administrative Science Quarterly v39, n4 (Dec 1994) pp.541-574 [15]Palmer, J. W. and Eriksen, L. B., "Digital Newspapers Explore Marketing on the Internet", Communications of the $A C M, \mathrm{v} 42, \mathrm{n} 9$, September 1999, pp. 33-40

[16]Peterson, R., Balasubramanian, S. and B. Bronnenberg, "Exploring the Implications of the Internet for Consumer Marketing" Journal of the Academy of Marketing Science, v25, n4 (1997): 329-346

[17]Quelch, J. and L. Klein, "The Internet and International Marketing” Sloan Management Review, 37, Spring, 60-75

[18]Raj, B. and B. Baltagi, eds. "Panel Data Analysis". Heidelberg: Physica-Verlag, 1992

[19]Rayport, J. and J. Sviokla, "Exploiting the Virtual Value Chain" Harvard Business Review, Nov-Dec 1995

[20]Stephenson, W. "The Play Theory of Mass Communication" Chicago: The University of Chicago Press, 1967

[21]Watters, C. R., M. A. Shepherd and F. J. Burkowski, "Electronic News Delivery Project". Journal of the American Society for Information Science v49, n2 (Feb 1998), pp. 134-150 [22]Weill, G. "El Diario; Historia y Función de la Prensa Periodica" México, Fondo de Cultura Económica, 1941

[23]Yates, J. and W. Orlikowsky "Genres of Organizational Communication: A Structurational Approach to Studying Communication and Media" Academy of Management Review. 17 (1992) pp. 299-

[24]Zellner, Arnold "An Efficient Method of Estimating Seemingly Unrelated Regressions and Tests of Aggregation Bias," Journal of the American Statistical Association, 57, (1962), pp. 348-368. 